OSU-HEP-98-2

UMD-PP-98-98

March 1998

\title{
A Four-Neutrino Mixing Scheme for Observed Neutrino Data
}

\author{
S. C. Gibbons ${ }^{1}$, R. N. Mohapatra ${ }^{2}$, S. Nandi ${ }^{1}$ and Amitava \\ Raychaudhuri ${ }^{1,3}$ \\ ${ }^{1}$ Department of Physics, Oklahoma State University, \\ Stillwater, Oklahoma 74078, USA \\ ${ }^{2}$ Department of Physics, University of Maryland, \\ College Park, Maryland 20\%42, USA \\ ${ }^{3}$ Department of Physics, University of Calcutta, \\ 92 Acharya Prafulla Chandra Road, Calcutta 700009, INDIA.
}

\begin{abstract}
It has been observed that simultaneous explanation of the solar and atmospheric neutrino deficits and the reported evidence for $\nu_{\mu} \rightarrow \nu_{e}$ oscillation from the Los Alamos Liquid Scintillator Detector (LSND) requires at least one extra neutrino species in addition to the three known ones. The extra neutrino must be sterile with respect to the known weak interactions. We present a new mass matrix for these four neutrinos in which the LSND effect and the atmospheric neutrino deficit are governed by only one parameter. We investigate the phenomenological implications of such a mass matrix ansatz and suggest possible ways to understand it in gauge theories.
\end{abstract}




\section{Introduction}

These are very exciting times in neutrino physics. For the first time in its history, there are several hints for a nonvanishing mass for at least two of three known neutrinos which look very promising and credible. They come (i) from the observation of the solar neutrinos in various experiments and their disagreement with the predictions of the standard solar model [1]: the earlier experiments from Homestake, Kamiokande, SAGE and GALLEX 2] and the most recent high statistics confirmation of these results by the super-Kamiokande experiment[3] and (ii) from

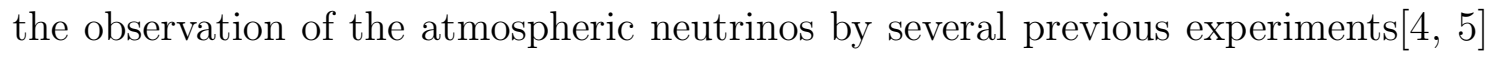
and the most recent confirmation of the earlier results by the super-Kamiokande[3] collaboration. Then there is the result from the Los Alamos Liquid Scintillator Neutrino Detector (LSND) which gives the first laboratory evidence for the oscillation of both $\bar{\nu}_{\mu} \rightarrow \bar{\nu}_{e}\left[\right.$ [6] as well as $\nu_{\mu} \rightarrow \nu_{e}$ type [7]. The existence of nonvanishing neutrino masses can be inferred from these data once one tries to understand them in terms of neutrino oscillations which depend on the mass difference square between the neutrino species (i.e. $\delta m_{i j}^{2}$ ).

It has been pointed out that since the above processes require three different $\delta m^{2}$, it is impossible to understand them within the conventional three neutrino $\left(\nu_{e}, \nu_{\mu}, \nu_{\tau}\right)$ framework; rather, one has to include a sterile neutrino which is ultralight (denoted by $\nu_{s}$ ) [8, 9]. To see this in detail, note that the MSW 10 type solution to the solar neutrino puzzle including the latest super-Kamiokande results yields the following ranges (the so-called small angle solution) for the neutrino mass differences and mixing [11],

$$
\delta m_{e i}^{2} \sim 4 \times 10^{-6}-1.2 \times 10^{-5} \mathrm{eV}^{2}, \sin ^{2} 2 \theta_{e i} \sim 3 \times 10^{-3}-1.5 \times 10^{-2}
$$

Next, a best fit to the presently available atmospheric neutrino data from Kamiokande, IMB and Soudan as well as super-Kamiokande assuming $\nu_{\mu}-\nu_{\tau}$ oscillation seems 
to require 12 at $90 \% \mathrm{CL}$ :

$$
4 \times 10^{-4} \mathrm{eV}^{2} \leq \delta m_{a t m}^{2} \leq 5 \times 10^{-3} \mathrm{eV}^{2}, \sin ^{2} 2 \theta_{a t m} \simeq .76-1
$$

Although the $\nu_{e}-\nu_{\mu}$ interpretation of this deficit for the older data from Kamiokande is ruled out by the recent CHOOZ[13] results, inclusion of the super-Kamiokande data still leaves an allowed domain for $\delta m_{e \mu}^{2}$. In this paper, we will assume $\nu_{\mu}-\nu_{\tau}$ oscillation to be the explanation of the atmospheric neutrino puzzle. Finally, the LSND data along with the constraints from the reactor experiment at Bugey 14 and E776 at BNL[15] suggest that:

$$
.2 \mathrm{eV}^{2} \leq \delta m_{e \mu}^{2} \leq 3 \mathrm{eV}^{2}, 2 \times 10^{-3} \leq \sin ^{2} 2 \theta_{e \mu} \leq 4 \times 10^{-2}
$$

We thus see that three different $\delta m^{2}$ values are needed and hence the obvious need for an extra sterile neutrino species.

Once one accepts the idea of four-way neutrino oscillation as a necessity to solve the neutrino puzzles, the next question is what is the nature of the mass matrix that can accommodate the known data and finally what it implies for physics beyond the standard model. It is the goal of this work to discuss the first question followed by an attempt to study the resulting theoretical implications.

\section{The four neutrino mass matrix}

A simple mass matrix ansatz that embodies the above mixing pattern was suggested in [16, 17] and analyzed in detail in [17]. This is given in the basis $\left(\nu_{s}, \nu_{e}, \nu_{\mu}, \nu_{\tau}\right)$ by:

$$
M=\left(\begin{array}{cccc}
\mu_{1} & \mu_{3} & 0 & 0 \\
\mu_{3} & 0 & 0 & a \\
0 & 0 & \delta & m \\
0 & a & m & \delta
\end{array}\right)
$$

where one requires that $\mu_{3} \ll \mu_{1}, \delta \ll a \ll m$. A characteristic of this mass matrix (to be called henceforth MI) is that the atmospheric neutrino oscillation is governed 
by the parameter $\delta$ whereas the mixing angle in the $\nu_{e}-\nu_{\mu}$ oscillation is given by the parameter $a$. They are unrelated.

Our proposal in this paper is to choose instead the following mass matrix:

$$
M=\left(\begin{array}{cccc}
\mu_{1} & \mu_{3} & 0 & 0 \\
\mu_{3} & 0 & 0 & a \\
0 & 0 & \delta & m \\
0 & a & m & -\delta
\end{array}\right)
$$

This mass matrix is the same as the one in eq. (雨) except for the negative sign in the $\nu_{\tau}-\nu_{\tau}$ entry. We will call this MII. We will see that this simple change of sign has profound phenomenological consequences. In particular, it implies that the same parameter $a$ is responsible for nonvanishing effects both for LSND as well as atmospheric neutrinos.

In order to study the phenomenological implications of this mass matrix, let us obtain the eigenvalues and the mixing matrix. We will assume that the above mass matrices are defined in a basis in which the charged leptons are mass eigenstates.

We choose the following hierarchy among the parameters: $\mu_{3} \ll \mu_{1} \ll a \ll$ $\delta, m$. Note already a difference from the mass texture MI that $\delta$ is much larger. To obtain the two largest eigenvalues, we ignore the small parameters $\mu_{1,3}$ and $a$. It is then clear that the two eigenvalues are equal in magnitude and opposite in sign i.e. $\lambda_{3,4}= \pm \sqrt{m^{2}+\delta^{2}}$. This, of course, is not of physical interest since it does not lead to atmospheric neutrino oscillation. However, it gives us an idea of how big the parameters $m$ and $\delta$ should be. For instance if $\nu_{\mu, \tau}$ are to constitute the hot dark matter of the universe, then $\sqrt{m^{2}+\delta^{2}}$ should be in the $\mathrm{eV}$ range.

To get the mass splitting between the $\nu_{\mu}$ and $\nu_{\tau}$ (mass eigenstates denoted by $\nu_{3}$ and $\nu_{4}$ respectively), we clearly have to keep the $a$ and consider the $3 \times 3$ matrix. 
Diagonalizing it, we find that the masses of $\nu_{\mu, \tau}$ are now split with

$$
m_{3}^{2}-m_{4}^{2} \equiv \delta m_{a t m}^{2} \simeq \frac{a^{2} \delta}{\sqrt{\delta^{2}+m^{2}+a^{2}}}
$$

Furthermore, the LSND $\nu_{e}-\nu_{\mu}$ mixing angle is given (see eq. (11]) by:

$$
\theta_{e \mu} \simeq \frac{a m}{m^{2}+\delta^{2}}
$$

We thus see that in MII, the single parameter $a$ is responsible for the atmospheric mass splitting as well as the LSND $\nu_{e}-\nu_{\mu}$ mixing angle. This is different from what happens in the MI case. We can therefore conclude that if $\nu_{e}-\nu_{\mu}$ oscillation becomes very small and if the $\delta m^{2}$ inferred from the atmospheric neutrino deficit remains where it is, MII will be ruled out. In fact, an even stronger statement can be made i.e. if one takes the $\delta m^{2}$ values from the LSND experiment consistent with E776 and Bugey (see eq. (3)), one has $0.02 \leq \theta_{e \mu} \leq .1$. From eqs. (6) and (7) $\delta m_{a t m}^{2} \simeq \theta_{e \mu}^{2} m \delta\left(\frac{m^{2}+\delta^{2}}{m^{2}}\right)^{\frac{3}{2}}$. This would at most be $1.3 \times 10^{-3} \mathrm{eV}^{2}$, implying that a significant part of the allowed $\delta m_{a t m}^{2}$ will be inconsistent with this model. In other words, as the atmospheric neutrino data becomes more precise MII will be testable unlike the MI texture where by adjusting the different parameters $\delta$ and $a$ a much larger range of values for the above experimental observables can be accommodated.

Let us now turn to the $\nu_{e}-\nu_{s}$ mixing which is responsible for understanding the solar neutrino puzzle in MII. For this purpose, we now consider the full $4 \times 4$ mass matrix and find for the $\nu_{e}$ and $\nu_{s}$ (mass eigenstates denoted by $\nu_{2}$ and $\nu_{1}$ respectively):

$$
m_{1}^{2}-m_{2}^{2} \equiv \delta m_{\text {solar }}^{2} \simeq\left(\mu_{1}+\frac{a^{2} \delta}{m^{2}+\delta^{2}}\right) \sqrt{\left(\mu_{1}-\frac{a^{2} \delta}{m^{2}+\delta^{2}}\right)^{2}+4 \mu_{3}^{2}}
$$

To address the solar neutrino puzzle via the MSW mechanism it is further necessary for the sterile neutrino to be heavier than the $\nu_{e}$. 
In order to discuss further tests of the model, we write the complete mixing matrix among the four neutrinos in the following form:

$$
U=\left(\begin{array}{cccc}
1 & \epsilon_{1} & 0 & 0 \\
-\epsilon_{1} & 1 & \epsilon_{2} & \epsilon_{3} \\
0 & \epsilon_{4} & c & s \\
0 & \epsilon_{5} & -s & c
\end{array}\right)
$$

where

$$
\begin{gathered}
s \simeq-\sqrt{\frac{C-\delta}{2 C}} ; \quad c \simeq \sqrt{\frac{C+\delta}{2 C}} ; \\
\epsilon_{1} \simeq \frac{-\mu_{3}}{\mu_{1}-\frac{a^{2} \delta}{m^{2}+\delta^{2}}} ; \quad \epsilon_{2} \simeq \frac{a}{C} \sqrt{\frac{C-\delta}{2 C}} \\
\epsilon_{3} \simeq-\frac{a}{C} \sqrt{\frac{C+\delta}{2 C}} ; \quad \epsilon_{4} \simeq-\frac{a m}{C^{2}} ; \quad \epsilon_{5} \simeq \frac{a \delta}{C^{2}}
\end{gathered}
$$

and $C=\sqrt{m^{2}+\delta^{2}}=\sqrt{\delta m_{e \mu}^{2}}$. Note that $C$ and $c$ refer to different quantities.

In terms of these mixings, we can write down the oscillation probabilities for various neutrino species $P\left(\nu_{i} \rightarrow \nu_{j}\right)$ as follows:

$$
\begin{aligned}
& P\left(\nu_{e} \rightarrow \nu_{e}\right) \simeq 1-4 \epsilon_{1}^{2} \sin ^{2} \Delta_{s}-4\left(\epsilon_{2}^{2}+\epsilon_{3}^{2}\right) \sin ^{2} \Delta \\
& P\left(\nu_{\mu} \rightarrow \nu_{\tau}\right) \simeq 4 c^{2} s^{2} \sin ^{2} \Delta_{43} \\
& P\left(\nu_{e} \rightarrow \nu_{\mu}\right) \simeq 4 c s \epsilon_{2} \epsilon_{3} \sin ^{2} \Delta_{43}-4 \epsilon_{4}\left(c \epsilon_{2}+s \epsilon_{3}\right) \sin ^{2} \Delta \\
& P\left(\nu_{e} \rightarrow \nu_{s}\right) \simeq 4 \epsilon_{1}^{2} \sin ^{2} \Delta_{21} \\
& P\left(\nu_{e} \rightarrow \nu_{\tau}\right) \simeq-4\left[-\epsilon_{2} \epsilon_{3} c s \sin ^{2} \Delta_{43}+\epsilon_{5}\left(-s \epsilon_{2}+c \epsilon_{3}\right) \sin ^{2} \Delta\right] \\
& P\left(\nu_{\mu} \rightarrow \nu_{s}\right)=0
\end{aligned}
$$

where we have defined $\Delta_{i j} \equiv \frac{1.27 \delta m_{i j}^{2} L}{E}$ with four of the $\Delta_{i j}$ equal, i.e. $\Delta_{42}=\Delta_{41}=$ $\Delta_{32}=\Delta_{31} \equiv \Delta$. The remaining two $\Delta_{i j}$ are very different and explicitly included. We have used the usual units i.e. $\delta m^{2}$ is in $\mathrm{eV}^{2} ; L$ is in kilometers and $E$ is in $\mathrm{GeV}$. 
The allowed ranges of the parameters where all constraints are satisfied are presented in Table 1.

\begin{tabular}{|c||c|}
\hline Parameter & Allowed Range \\
\hline$m$ & $0.39 \mathrm{eV}-1.73 \mathrm{eV}$ \\
\hline$\delta$ & $2.2 \times 10^{-3} \mathrm{eV}-0.85 \mathrm{eV}$ \\
\hline$a$ & $2.0 \times 10^{-2} \mathrm{eV}-0.2 \mathrm{eV}$ \\
\hline$\mu_{1}$ & $5.5 \times 10^{-3} \mathrm{eV}-6.5 \times 10^{-3} \mathrm{eV}$ \\
\hline$\mu_{3}$ & $9.0 \times 10^{-6} \mathrm{eV}-1.3 \times 10^{-4} \mathrm{eV}$ \\
\hline
\end{tabular}

Table 1: The allowed ranges for the parameters of the model consistent with present accelerator, reactor, solar and atmospheric neutrino results.

These ranges must be taken together with the constraints:

$$
\begin{gathered}
m=C \sin \left(2 \theta_{a t m}\right) ; \quad \delta=C \cos \left(2 \theta_{a t m}\right) ; \quad \frac{a^{2} \delta}{C}=\frac{\delta m_{a t m}^{2}}{2} \\
3.3 \times 10^{-4} \leq \frac{\mu_{1} C^{2}-a^{2} \delta}{C^{2}} \leq 2.1 \times 10^{-3}
\end{gathered}
$$

The ranges in Table 1 and the above constraints are based on the assumption that $\mu_{1}$ is positive. If $\mu_{1}$ is negative then (a) the constraint (13) above does not apply, (b) Table 1 refers to the modulus of $\mu_{1}$, and (c) the range for $\mu_{3}$ changes to $1.5 \times 10^{-4} \leq$ $\mu_{3} \leq 7.4 \times 10^{-4}$

To summarize this section, we note that in our mass matrix we use five parameters to accommodate six experimental numbers i.e. three mass difference squares and three mixing angles for the solar, atmospheric and LSND experiments subject to the constraints from the Bugey and E776 results. (We have checked that the results from the CHOOZ and Fermilab E531 18 experiments are automatically satisfied when the parameters are in the allowed ranges.) More importantly, because of the intimate link between the $\theta_{e \mu}$ and $\delta m_{\text {atm }}^{2}$, our mass texture MII is more easily testable than the MI texture. 


\section{Other experimental tests}

Turning now to the tests of the model, the first well known point to emphasize is that the $\nu_{e} \rightarrow \nu_{s}$ oscillation solution to the solar neutrino problem will be tested once the SNO measures the neutral current effects of the solar neutrinos. Unlike the $\nu_{e} \rightarrow \nu_{\mu}$ oscillation scenario, where one would expect $\Phi_{C C} / \Phi_{N C} \simeq .4$, for the $\nu_{e} \rightarrow \nu_{s}$ case, one should get $\Phi_{C C} / \Phi_{N C} \simeq 1$. Here $\Phi$ represents the signal for the appropriate process before the incorporation of the cross-sections and detection efficiencies.

The second well known test of the model is of course in the long baseline search for $\nu_{\mu} \rightarrow \nu_{\tau}$ oscillation in experiments such as MINOS, where the oscillation probability is driven by $\Delta_{a t m}$ and we expect

$$
\Delta_{a t m} \simeq\left(\frac{\delta m_{a t m}^{2}}{5 \times 10^{-3} \mathrm{eV}^{2}}\right)\left(\frac{L}{157 \mathrm{~km}}\right)\left(\frac{\mathrm{GeV}}{E}\right)
$$

For $E=10 \mathrm{GeV}$, for the MINOS experiment this would imply a transition probability of about $25 \%$ for $\delta m^{2} \simeq 5 \times 10^{-3} \mathrm{eV}^{2}$.

Let us now turn to $\nu_{e} \rightarrow \nu_{\tau}$ oscillation. In this case, there is a difference between the MI and the MII scenarios. In both scenarios there are two mass scales that contribute to this probability: the one that governs the atmospheric neutrino deficit and the other which is associated with the LSND effect. However, from eqs. (10, 11) it is obvious that in the MII scenario the contribution of the $\sin ^{2} \Delta$ term is considerably larger than in the MI case where $\delta \ll m$. Taking values for $\epsilon_{2} \simeq .02$, $\epsilon_{3} \simeq-.03$, and $\epsilon_{5} \simeq .03$ as dictated by our choice of parameters given earlier, we expect that in a long baseline experiment,

$$
P\left(\nu_{e} \rightarrow \nu_{\tau}\right) \simeq 3 \times 10^{-4}+10^{-3} \sin ^{2} \Delta_{a t m}
$$

In the MI scenario, the first term is negligible.

The predicted $\nu_{e} \rightarrow \nu_{\tau}$ oscillations driven by two vastly different mass-scales but of comparable strength could be observable in future facilities (presently under 
contemplation) such as the muon collider with a long baseline neutrino experiment [19]. For the purpose of illustration we consider the detectability of a $\nu_{\tau}$ signal through the $\tau^{-} \rightarrow \mu^{-}$decay where the parent $\nu_{e}$ beam of $E_{\max }=20 \mathrm{GeV}$ originates from the decay of $\mu^{+}$s produced for a muon collider set-up at Fermilab. Our results are presented in the Figure. We find that a $10 \mathrm{kT}$ detector at Soudan will be able to scan, at the single event/year signal level, significant portions of the predicted mixing angle - mass-square-difference space of both the mass scales but Gran Sasso will be sensitive to a smaller part of only the LSND mass-scale. If a $10 \mathrm{kT}$ detector could be set-up at a distance of $10 \mathrm{~km}$ from the source then its reach in the mixing angle will be much wider and we show in the figure the sensitivity of such a facility assuming 100 signal events/year.

There will also be testable difference between the MI and the MII scenarios in the short baseline experiments. In this case, the $\Delta_{34}$ contribution to $P\left(\nu_{e} \rightarrow\right.$ $\nu_{\tau}$ ) would be insignifcant. As a result, in the MI scenario, one would expect this oscillation to be negligible. On the other hand in the MII scenario, we get

$$
P\left(\nu_{e} \rightarrow \nu_{\tau}\right) \simeq 10^{-3} \sin ^{2} \Delta
$$

This is large enough to be observable with a reasonable $\tau$ detection efficiency.

In our model, the neutrinoless double beta decay vanishes at the tree level since its amplitude is proportional to $\left\langle m_{\nu_{e}}>\right.$ which is given by

$$
<m_{\nu_{e}}>=\Sigma_{i} U_{e i}^{2} \eta_{i} m_{\nu_{i}}=M_{\nu_{e} \nu_{e}}
$$

Here $M_{\nu_{e} \nu_{e}}$ is the $\nu_{e}-\nu_{e}$ element of the neutrino mass matrix in the weak basis and $\eta_{i}= \pm 1$ is the sign of the $i$-th eigenvalue. As is clear from eq. (5), this entry vanishes in our case. This also happens in the scenario MI, contrary to the assertion in Ref. [17]. 


\section{Possible theoretical scenarios}

In this section, we explore possible theoretical scenarios that could lead to the mass texture analyzed in this paper. Specifically, we want to reproduce the key distinguishing feature of the model which is $M_{\nu_{\mu} \nu_{\mu}}=-M_{\nu_{\tau} \nu_{\tau}}$ within a reasonable scenario that can also roughly account for the rest of the texture.

Let us consider an extension of the standard model where the fermion sector is left untouched but the Higgs sector has several additional scalars and the theory is invariant under the extra global symmetry $S_{3} \times Z_{2}$ where $S_{3}$ is the permutation symmetry on three objects. The extra Higgs fields included are two triplets $\left(\Delta_{i}\right)$ with lepton number $L=-2$, which acquire a tiny vev due to a Higgs see-saw mechanism; four doublets $H_{1,2}$ and $H_{3,4}$ with identical quantum numbers to the standard model doublet and different quantum numbers under the discrete global symmetry group; two iso-singlet charged $\left(\eta_{i}^{+}\right)$fields with $L=+2$; a doubly charged iso-singlet field $\left(k^{++}\right)$with $L=-2$ and a neutral $L=2$ field $\sigma$. We will assume that $\left.\left.\langle\sigma\rangle \gg<H_{2,3,4}^{0}\right\rangle \gg<\Delta_{i}^{0}\right\rangle \simeq 1-2 \mathrm{eV}$. We further assume that $\left\langle H_{1}^{0}\right\rangle=0$. The $S_{3} \times Z_{2}$ transformation property of the Higgs and fermion fields are presented in Table 2.

\begin{tabular}{|c|c|c|c|c|c|c|c|c|c|c|}
\hline Fields & $\left(\begin{array}{c}L_{\mu} \\
L_{\tau}\end{array}\right)$ & $L_{e}$ & $\left(\begin{array}{c}\mu^{c} \\
\tau^{c}\end{array}\right)$ & $e^{c}$ & $H_{3,4}$ & $\left(\begin{array}{c}H_{1} \\
H_{2}\end{array}\right)$ & $\left(\begin{array}{c}\Delta_{1} \\
\Delta_{2}\end{array}\right)$ & $k^{++}$ & $\left(\begin{array}{l}\eta_{1} \\
\eta_{2}\end{array}\right)$ & $\sigma$ \\
\hline $\begin{array}{c}S_{3} \times Z_{2} \\
\text { quantum } \\
\text { numbers }\end{array}$ & $(2,+)$ & $(1,-)$ & $(2,+)$ & $(1,-)$ & $(1,+,-)$ & $(2,+)$ & $(2,+)$ & $(1,+)$ & $(2,-)$ & $(1,+)$ \\
\hline
\end{tabular}

Table 2: The lepton and scalar fields of the model and their $S_{3} \times Z_{2}$ quantum numbers. There are two one-dimensional representations of $S_{3}$. Here 1 stands for the invariant representation. 
The Yukawa Lagrangian invariant under the gauge group and the extra global symmetries is:

$$
\begin{aligned}
\mathcal{L}= & f_{1}\left[2 L_{\tau} L_{\mu} \Delta_{1}+\left(L_{\mu} L_{\mu}-L_{\tau} L_{\tau}\right) \Delta_{2}\right] \\
& +h_{1} L_{e} H_{3} e^{c}+h_{2}\left(L_{\mu} \mu^{c}+L_{\tau} \tau^{c}\right) H_{3} \\
& +h_{3}\left[\left(L_{\mu} \tau^{c}+L_{\tau} \mu^{c}\right) H_{1}+\left(L_{\mu} \mu^{c}-L_{\tau} \tau^{c}\right) H_{2}\right] \\
& +f_{2}\left(L_{\tau} \eta_{2}+L_{\mu} \eta_{1}\right) L_{e}+f_{3}\left(\tau^{c} \tau^{c}+\mu^{c} \mu^{c}\right) k^{--}+f_{4} e^{c} e^{c} k^{--}
\end{aligned}
$$

The Higgs Lagrangian will contain two parts: one part consisting only of dimension four operators that are invariant under the gauge as well as the global symmetry and a part consisting of terms with dimension two or three which will softly break the global symmetries. In the equation below, we give only the part of the potential relevant to generating the neutrino Majorana masses out of two loop effects.

$$
\begin{aligned}
& \mathcal{L}_{\text {Higgs }}=V_{0}+\mu_{1}\left({\eta_{1}^{+}}^{2}+\eta_{2}^{+2}\right) k^{--}+\lambda\left(\Delta_{1} H_{1}+\Delta_{2} H_{2}\right) H_{3} \sigma+m_{0}^{2} \Delta_{1}^{\dagger} \Delta_{2} \\
& +\lambda^{\prime} H_{4}\left[\left(\Delta_{1} H_{2}+\Delta_{2} H_{1}\right) \eta_{1}+\left(\Delta_{1} H_{1}-\Delta_{2} H_{2}\right) \eta_{2}\right]+\text { h.c. }
\end{aligned}
$$

Let us now study the tree level mass matrix for the three known neutrinos in this model. It is given by

$$
M_{0}=\left(\begin{array}{ccc}
0 & 0 & 0 \\
0 & f_{1} v_{2} & f_{1} v_{1} \\
0 & f_{1} v_{1} & -f_{1} v_{2}
\end{array}\right)
$$

where $v_{i}=<\Delta_{i}^{0}>$. Thus this has the desired structure for the scenario MII in the $\nu_{\mu}-\nu_{\tau}$ sector. Incidentally, the $\lambda$ term in the Higgs Lagrangian can be used to suppress the $\Delta_{i}$ vev's via a see-saw mechanism.

The $\nu_{e}-\nu_{\tau}$ entry (i.e. $a$ ) in the mass matrix arises via a two loop diagram. The magnitude for this term can be estimated to be

$$
a \simeq \frac{f^{3} \mu^{3} m_{\tau}^{2}}{\left(16 \pi^{2}\right)^{2} M^{4}}
$$


where $M$ is a typical scalar mass. If we choose $f_{i} \approx 4 \times 10^{-3}$ and $\mu \approx 10 M \simeq 1 \mathrm{TeV}$, then we get $a \approx .01 \mathrm{eV}$ which is of the right order of magnitude. We have checked that the contributions to other entries of the neutrino mass matrix are adequately small. It is important to remark that for our mixing considerations to hold, the charged leptons must be diagonal. This is guaranteed by $<H_{1}>=0$. We have to fine tune $h_{2}<H_{3}>$ and $h_{3}<H_{2}>$ to get the muon and the tau lepton masses right.

So far we have ignored the sterile neutrino. It can be incorporated into the model in one of two ways. An elegant way to do this is to double the particle spectrum and the gauge forces of the standard model by including a mirror sector to the standard model as is done in Ref. 20] and inducing the Majorana masses for the neutrinos via non-renormalizable operators. The lightest neutrino of the mirror sector will be identified with the sterile neutrino. The non-renormalizable operators will in general induce nonzero values in the $\nu_{e}-\nu_{\mu}$ entry as well as in the $\nu_{e}-\nu_{e}$ entry but these contributions are in the range of $10^{-6} \mathrm{eV}$ so that they do not affect any of our considerations. The mirror $\nu_{\mu, \tau}^{\prime}$ masses will arise from triplets whose vevs will be larger than the corresponding ones in the visible sector since the see-saw mechanism giving their vevs is proportional to doublet vevs, which for cosmological reasons must be larger in the mirror sector: $\left\langle H_{\text {mirror }}>\simeq 30<H>\right.$.

Another way is to add the sterile neutrino as an extra fermion to the standard model and use the radiative mechanism to suppress its mass to the desired level. By adding new Higgs fields to the model already described, this can be accomplished. We do not elaborate on this here.

\section{Conclusion}

In conclusion, we have presented a new four neutrino mass matrix which fits all neutrino observations with five parameters and where the $\nu_{e}-\nu_{\mu}$ mixing and 
the $\delta m_{\mu \tau}^{2}$ are related to each other unlike the simplest four neutrino mass matrix discussed in the literature. Thus, in this scenario the atmospheric neutrino deficit seen in the present data implies the observable effect in $\nu_{\mu}-\nu_{e}$ oscillation as found at LSND. This model can also be tested in the short as well as long baseline $\nu_{e}-\nu_{\tau}$ oscillation searches. We also construct a theoretical scenario which could lead to the mass matrix of the type under consideration.

\section{Acknowledgement}

The work of S. C. G., S. N., and A. R. is supported by the U.S. Department of Energy while that of R. N. M. is supported by a grant from the National Science Foundation, grant no.PHY-9421385. A. R. is also partially supported by grants from C.S.I.R., India and D.S.T., India. He is grateful to the Department of Physics of the Oklahoma State University for their hospitality.

\section{References}

[1] J. Bahcall, Proceedings of Neutrino'96 edited by K. Enquist, K. Huitu and J. Maalampi (World Scientific, Singapore); A. Smirnov, in Warsaw 1996, ICHEP 96, Vol. 1 p288 (hep-ph/9611465).

[2] B. T. Cleveland et al. Nucl. Phys. B (Proc. Suppl.) 38 (1995) 47; K. S. Hirata et al., Phys. Rev. D44 (1991) 2241; GALLEX Collaboration, Phys. Lett. B388 (1996) 384; J. N. Abdurashitov et al., Phys. Rev. Lett. 77 (1996) 4708.

[3] Y. Suzuki, Invited talk at Erice Neutrino workshop, September 17-22, 1997.

[4] K.S. Hirata et al., Phys. Lett. B280 (1992) 146; R. Becker-Szendy et al., Phys. Rev. D46 (1992) 3720; W. W. M. Allison et al., Phys. Lett. B391 (1997) 491.

[5] Y. Fukuda et al., Phys. Lett. B335 (1994) 237. 
[6] C. Athanassopoulos et al. Phys. Rev. Lett. 75 (1995) 2650.

[7] C. Athanassopoulos et al. nucl-ex/9706006.

[8] D. O. Caldwell and R. N. Mohapatra, Phys. Rev. D48 (1993) 3259; J. Peltoniemi and J. W. F. Valle, Nucl. Phys. B406 (1993) 409.

[9] S. Goswami, Phys. Rev. D55 (1997) 2931; S. M. Bilenky, C. Giunti and W. Grimus, Eur. Phys. J. C1 (1998) 247; hep-ph/9711311.

[10] L. Wolfenstein, Phys. Rev. D17 (1978) 2369; S. P. Mikheyev and A. Smirnov, Yad. Fiz. 42 (1985) 1441; Nuovo Cimento, 9C (1986) 17.

[11] G. L. Fogli, E. Lisi and D. Montanino, hep-ph/9709473.

[12] M. C. Gonzalez-Garcia, et al., hep-ph/9801368.

[13] M. Apollonio et al. hep-ex/9711002.

[14] B. Achkar et al., Nucl. Phys. B434 (1995) 503.

[15] L. Borodovsky et al., Phys. Rev. Lett. 68 (1992) 274.

[16] R. N. Mohapatra, hep-ph/9711444.

[17] V. Barger, T. Weiler and K. Whisnant, hep-ph/9712495.

[18] N. Ushida, et al., Phys. Rev. Lett. 57 (1986) 2897.

[19] S. Geer, hep-ph/9712290.

[20] Z. Berezhiani and R. N. Mohapatra, Phys. Rev. D52 (1995) 6607. 


\section{FIGURE CAPTION}

The prediction of this model for $\nu_{e}-\nu_{\tau}$ oscillation in an effective two-flavor plot is shown as the rectangular regions. The sensitivity of experiments located at Gran Sasso $(\mathrm{L}=9900 \mathrm{~km})$, Soudan $(\mathrm{L}=732 \mathrm{~km})$ and a new facility $(\mathrm{L}=10 \mathrm{~km})$ are shown when the source is a $\nu_{e}$ beam obtained from a parent muon beam (see text) at Fermilab with $E_{\max }=20 \mathrm{GeV}$. The Gran Sasso and Soudan results correspond to a single-event signal/year while for the new facility at $10 \mathrm{~km}$ the results shown correspond to 100 signal events/year. 


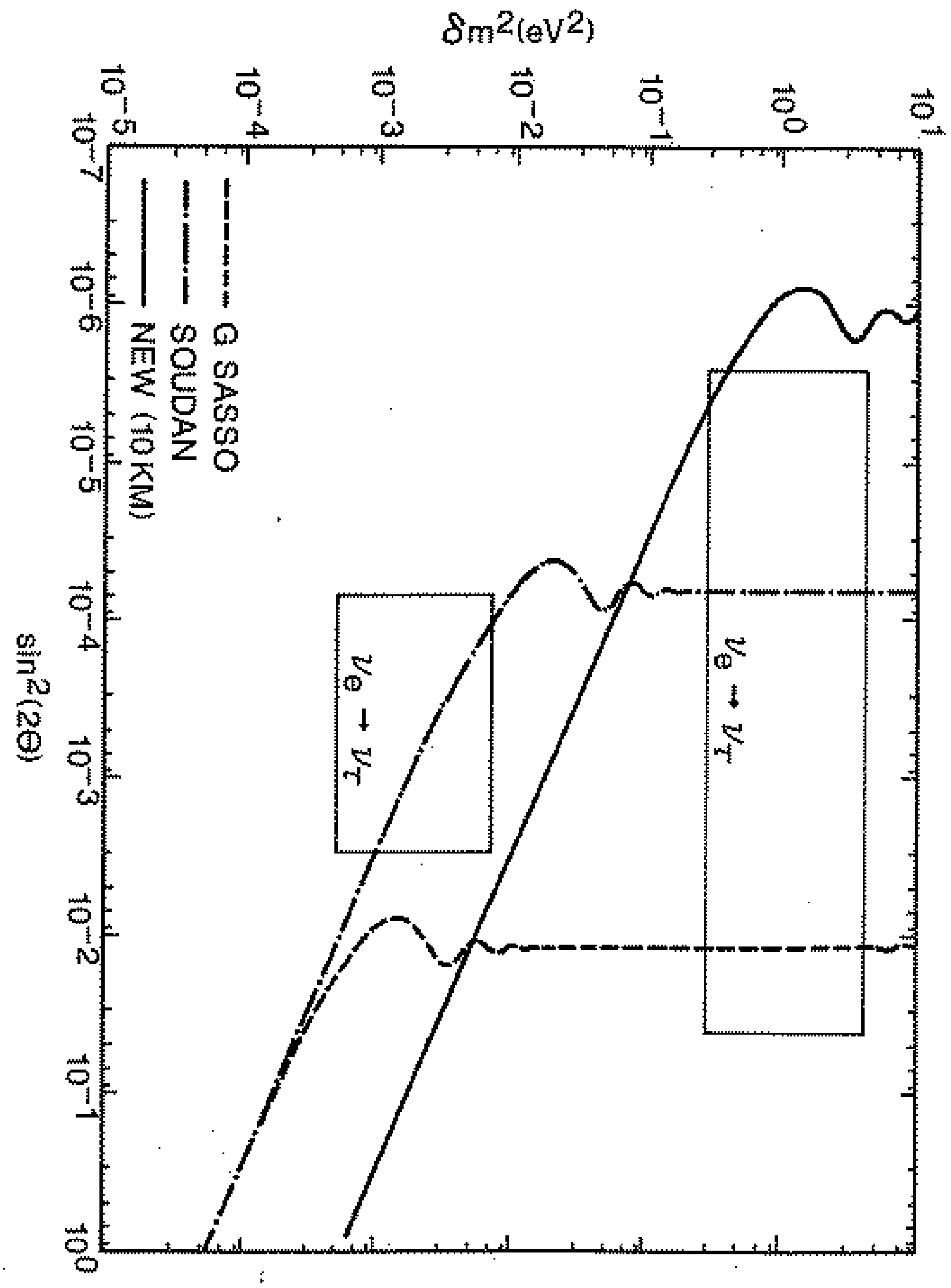

